$\xi=-$ 圆

\title{
Support vector machine the most fruitful algorithm for prognosticating heart disorder
}

\author{
M. Murugesan ${ }^{1 *}$, R. Elankeerthana ${ }^{2}$ \\ ${ }^{1}$ Assistant Professor, Department of Computer Science and Engineering,M.Kumarasamy College of Engineering, India \\ ${ }^{2}$ M.E.Student, Department of Computer Science and Engineering, M.Kumarasamy College of Engineering, , India \\ *Corresponding author E-mail: elankeerthana25@gmail.com
}

\begin{abstract}
One of the wealthiest areas of research is Data mining that is more popular in healthcare organizations. Heart disease is the main outcome of death in the human society over the recent years. Heart disease is serious life threatening diseases that result to death. In order to save a pan-tient's life, the doctors and medical examiners are being taking many efforts. The consultant of doctor's determination can make without the advice of specialists because of the software develop by the advancement in computer technology. In most of the papers, Data mining tech-niques used in the existing method in the research are Naive Bayes, Decision tree, J48, K-Nearest Neighbor (K-NN) (or) Lazy IBK algo-rithms to predict heart diseases. In this paper, support vector machines (SVM) technique will produce the most accuracy prediction rate for heart diseases while comparing to all the other techniques used in data mining.
\end{abstract}

Keywords: Heart Disease; Data Mining; Decision Tree; J48; Naive Bayes; K-NN; Lazy IBK; Support Vector Machines.

\section{Introduction}

The process of analyzing data from dissimilar perspectives and resulting into helpful information that can be used to enhance revenue, cuts costs, or both is called Data Mining (knowledge spotting of database). It is major concerned with useful information is extracted from large quantity of databases. Correlations or a pattern among dozens of fields from relational databases is obtained from the process called Data mining. Unknown design and trends from the data set are determined from the Data mining techniques and tools. Minimal user effort and input in the dataset is automatically detected from the pattern is the major objective. Forecasting future trends of market and decision making are the major contribution in Data mining. These days, multiple organizations use data mining as a tool for data analysis to estimate current and design of market and yield effective outcomes. Data extracted from large quantities from automatic analysis or semi-automatic, interesting design such as unusual records (anomaly detection),cluster of data records (cluster analysis), and dependencies (association rule mining, sequential design mining) is the exact data mining task.

In Medical Application:

1) Medical records of billion people's about $3 / 4$ are available electronically.

2) Comparing to other fields, Data mining in the medical field is distinct due to nature of data: social constraints, heterogeneous with ethical and legal.

3) The data in the database are described by associative rules.

4) 4.Classification and prediction are most commonly used technique with different techniques used for different cases

5) The most rewarding even with the difficulty is Medical data mining.
Table 1: 10 Biggest Killers in the World

\begin{tabular}{cccc}
\multicolumn{4}{c}{ Table 1: 10 Biggest Killers in the World } \\
\hline CAUSE & DEATH & DRUGS & \$ BILLIONS \\
\hline Cardiology & 739,000 & Cardiovascular & 16.5 \\
Cancer & 538,000 & Anti-cancer & 4.1 \\
Crack or & 158,000 & Anti-coagulants & 0.3 \\
Stroke & & Respiratory & 4.4 \\
Lung Disor- & 105,000 & - & - \\
ders & 90,000 & Antibiotics/Antibac- & 9.5 \\
Accidents & 84,000 & terial & 0.8 \\
Pneumonia & Insulin & 0.3 \\
\& Flu & 59,000 & Antiviral & 4.6 \\
Diabetes & 43,000 & Antidepressants and & - \\
AIDS & 31,000 & Antipsychotics & - \\
Suicide & 25,000 & - & \\
Homicide & & & \\
\hline
\end{tabular}

From TABLE 1, it is shown the highest number of death is happened by heart diseases. It is necessary to detect in the early stages and being treated for the disease.

Therefore, we can predict heart diseases by various algorithms in Data mining field.

1) Modeling

Models such as Build, Data mining algorithm, Data mining use case, Data mining goal, Data mining data, Data mining and Data mining technique.

2) Data Mining Application

Financial Data Analysis, Intrusion Detection, Student's Performance and Sports, Industries such as Telecommunication, Healthcare, Retail.

3) Data Mining Techniques

Association, Clustering, Classification, Prediction,

SequentialPatterns, Regression,Summarization.

4) Data Mining

Tools: WEKA, TANAGRA, MATLAB,.NET FRAMEWORK. 
For people with CVD or high cardiovascular risk (blood vessels or the heart that involve in diseases, CVDs include stroke, arterial hypertension, diabetes, Hyperlipidemia, aortic aneurysms and others) is extremely important early detection and treatment. Currently, several studies have focused in cardiovascular disease diagnosis, with the ideal of predicting or detecting in advance the risks of taking some type of CVD. Especially for men, who died suddenly will have coronary disease with plaque morphology and Coronary Risk Factors. Current research has implemented several techniques or methods to achieve the best precision levels in classification, guaranteeing a reliable system to be used for decision making to diagnose in assertive manner the presence of CVD. Admission in the hospital or unnecessary treatment may undergo while Patient having heart pain complaint. Specialists for heart are not widely available for the diagnosis from the well developing countries. So, such automatic system will help community like medical to consult doctor for the exact diagnosis well in advance.several computational intelligence techniques are studied for heart disease detection, and their study is based in comparing six known classifiers with the data provided by the University of Cleveland. In that research the method with better accuracy percentage was support vector machines. Particularly, maximum and minimum of $86.36 \%$ and $45.45 \%$ will represented by both RBF kernel SVM and polynomial kernel SVM respectively.

Heart Attack Can Include Symptoms:

- Discomfort, stress, heaviness, or pain like clenched fist below the breastbone or in the chest.

- Discomfort radiating to the back, throat, jaw or arm.

- Fullness, burning sensation, or choking feeling (heartburn).

- Sweating, nausea, dizziness.

- Extreme fragility, anxiousness, fatigue.

- Rapid or irregular heartbeats.

- Shortness of breath.

- Neck, Arm, Chest(discomfort or tightness)

Heart Disease Can Include The Risk Factor:

- Family History of Heart Disease.

- Smoking, Tobacco.

- Cholesterol (fat).

- High Blood Pressure (HBP).

- Obesity.

- Diabetes.

- $\quad$ Lack of Physical Exercise.

- Eating Habits.

- Stress.

- Hypertension.

- Poor Diet.

\section{Literature survey}

Prediction of heart disease has been focused by several studies. Different data mining tools and applied various data mining techniques are used for diagnosis and achieved various conclusions. The goal of all is to achieve better accuracy and to make the system more efficient so that it can predict the risk of heart attack. Patients with high risk of having heart attack can be easily identified by the professionals with the help of health care associated with risk factor knowledge.

Fabio Mendoza Palechor* et al.[5] In 2016, 'Cardiovascular Disease Analysis Using Supervised and Unsupervised Data Mining Techniques' using data mining algorithms such as 'Bayesian networks ,decision trees, support vector machines(SVM) and k-nearest neighbors', where concluded Support Vector Machine (SVM) leaves high precision of $97 \%$.

K.Manimekalai [7]. In 2016, 'A Proficient Heart Disease Prediction Method Using Different Data Mining Tools' using tool of data mining for prediction of heart disease. They used BNN gives accuracy of $78.43 \%$ and sensitivity of $96.55 \%$.RBF kernel SVM represents specificity of $86.36 \%$.
Sharan Monica L et al.[12] In 2016, 'Analysis of Cardiovascular Heart Disease Prediction Using Data Mining Techniques' using tool of data mining for heart attack prediction. They used many algorithms which shows accuracy such as J48 is $91.4 \%$,NB Tree $88.5 \%$ and simple CART is $92.2 \%$.

H K Shifali et al.[6] In 2017, 'Mining of Medical Data to Identify Risk Factors of Heart Disease Using Frequent Itemset' using tool of data mining for detecting heart risk. They used Frequent itemsets and support value to generated the attributes used in Verdaderos Positive dataset by threshold $40 \%$ and $50 \%$ to know the risk.

Prof. Mamta Sharma et al. [9] In 2017,'Comparing Data Mining Techniques Used For Heart Disease Prediction' using tool of data mining to predict the heart stroke. They used algorithms which gives accuracy such as Navie Bayes gives $94.44 \%$ in 13 attributes and $90.74 \%$ in 15 attributes, Decision tree gives $96.66 \%$ in 13 attributes and $99.62 \%$ in 15 attributes and Neural Networks gives $99.25 \%$ in 13 attributes and $100 \%$ in 15 attributes.

Shashikant Ghumbre et al.[13] In 2011, ' Heart Disease Diagnosis using Support Vector Machine' using data mining tool to analysis the heart diseases. They used SVM algorithm and RBF network to identify the accuracy of heart attack such as $80.81 \%$ and $86.42 \%$ respectively based on overall average performance, $82.71 \%$ and $85.05 \%$ respectively based on performance using 5-Fold CrossValidation and $82.24 \%$ and $85.05 \%$ respectively based on performance using 10-Fold Cross-Validation[ Radial Basis Function(RBF) and Support Vector Machine (SVM)].

Era Singh Kajal et al.[4] In 2016, ' Prediction of Heart Disease using Data Mining Techniques' using tool of data mining to analysis the heart diseases. They used algorithms such as Decision tree, $\mathrm{K}$ $\mathrm{NN}$ and SVM to find accuracy from data set [K-Nearest Neighbor (K-NN) and Support Vector Machine (SVM)].

Akshaya et al.[2] In 2015, ' Data Mining Techniques to Diagnose Heart Diseases' using data mining tool to predict the cardio diseases. They proposed that Decision Tree and Support Vector Machine (SVM) are the most effective ones to evaluate the heart risk.

Ms S. Vijayarani et al.[15] In 2013, 'Comparative Analysis of Bayes and Lazy Classification Algorithms' using data mining tool for comparing Naive Bayes and Lazy IBK where the Naive Bayes gives precision as $79.30 \%$ and Lazy IBK gives precision as $89.90 \%$.[15]

\section{Problem statement}

Naive Bayes (NB):

NB has significant more elasticity of the logistic regression model without cost is not resulted. Though the forms such as resulting model and naive Bayes model is identical to one another (with dissimilar parameter values), it cannot be identified by seeing at the uni-variate marginal's separately: NB use an iterative procedure. K-nearest-neighbor (KNN) or IBK:

Drawback of KNN approach is obvious that they do not correctly match any of the training records because of that many test records will not be classified. The main difficult of KNN method is to set the value of $\mathrm{k}$, as if $\mathrm{k}$ takes a higher value than it should, the risk of classifying according to the majority of the data is too high, and weather the $\mathrm{k}$ value is too low, then comparison between the selection of few data's can make a delay of precision in classification instances.

\section{Methodology}

\subsection{Decision tree}

One of the most popular tools called Decision trees is non-parametric supervised learning mining technology. A decision tree is used for classification of dataset. Build a model that predicts the value of a aim variable by learning simple decision rules from dataset is the main aim of decision tree. The classification of instances by decision tree can be started from the root and moving through it until a leaf node of tree (top-down strategy). Production of a decision tree 
is an efficient method of generating easy-to interpret If-Then decision rule for fast construction. So, it has become the most widely used technique among different classification methods. Decision tree algorithm is used in medical tasks, such as improving status of dermatologic diagnosis, detecting essential hypertension and cardiovascular disease.

\subsection{Naive bayes}

The basis for data mining methods and numerous machine learning is Naive Bayes Rule. A single algorithm for training is Naive Bayes such as classifiers but it is also a group algorithms because of having some common rule- All Naive Bayes classifiers. Bayesian classifier is used to minimize the error rate. Continuous data set is used rather than the categorical data to predict. By structural, it has four levels. Creating models with predictive capabilities and creative ways of exploring data is also done by Naive Bayes. It understand from the "proof" by calculating the correlation between the target and other variables such as dependent and independent respectively. Therefore independent variables are taken for the purpose of prediction or occurrence of the event. Baye's Theorem determines the possibility of an even occurring given the possibility of another event that has already occurred. If B and A represents as the dependent event and the previous event, then the theorem can be stated as follows,

$\mathrm{P}(\mathrm{B}$ given $\mathrm{A})=\operatorname{Prob}(\mathrm{A}$ and $\mathrm{B}) / \operatorname{Prob}(\mathrm{A})$

To obtain the possibility of $\mathrm{B}$ given $\mathrm{A}$, the algorithm counts the number of cases where $\mathrm{A}$ and $\mathrm{B}$ occur $\mathrm{P}(\mathrm{Ci} / \mathrm{X})>\mathrm{P},(\mathrm{Cj} / \mathrm{X})$ for all $1<$ $=\mathrm{j}<=\mathrm{m}$ and $\mathrm{j} !=\mathrm{i}$

Naive Bayes algorithm is preferred in the following cases.

- Algorithm is used when the data has high dimensionality.

- There are self-governing attributes of each other. Otherwise, for simplification, assume the attributes to be independent to computations involved in the sense, is considered "naive".

- When we expect more efficient output, while comparing to other methods.

- Expose peak accuracy and speed while using in larger databases.

\subsection{J48}

In weka data mining tool, there is algorithm with $\mathrm{C} 4.5$ which is an open source java implementation is called J48 decision tree. Both continuous and discrete features are supported by J48. It can also manage features with missing value. Information which is collected from data set is used to take the decisions.J48 is the mathematical tool. In each tree node, which variable fits better in terms of target variable prediction is decide by $\mathrm{J} 48$.

\subsection{IBK (K - nearest neighbour)}

$\mathrm{K}-\mathrm{NN}$ is used for classification and data regression. A k-nearestneighbor classifier that uses the same distance metric is IBK.One of the first options for a categorization study when there is little or no prior knowledge about the distribution of data is K-NN. A group of $\mathrm{k}$ objects can be find by KNN classification in the training set that are nearest to the test object, and bases the assignment of a label on the predominance of a particular class in this neighborhood is the metropolitan approach. The main difficult of K-NN algorithm is to set the value of $k$, since if $k$ takes a higher value than it should, the risk of classifying according to the majority of the data is too high, and if the $\mathrm{k}$ value is too low, there can be a lack of precision in classification due to too few data selected as comparison instances. One of the most important benefit of this method is that can change radically its classification results without modifying its structure, the distance can be find by only changing the metric. The metric can be chosen by us. The advantage of KNN is you can obtain different results without transforming the algorithm of the method, only changes are made in the procedure of measuring distances.
In daily applications, one of the most strong and exact methods among all the algorithms is Support Vector Machines (SVM). Performing numerical calculations and regression analysis can be easily absolute by SVM, where estimating a linear function can approximate the target function is the goal. In the sample (training) data, SVM differentiating between the members of the two classes to estimate the best classification function.SVM offers the best generalization ability because of the best reasons called Hyperplane. It allows the most excellent classification presentation on the training data, and also gives more data for the exact categorization for the outlook use. Amount of space is called the margin and separation between the two classes is known by the hyperplane. Maximizing the margin between the two classes can found the best function that is guarantee by SVM. The maximum figure of training data where the function class can still be used to learn faultlessly, the VC dimension measures the training data for obtaining zero error rates. Training error is the first and second term whether proportional to the square root of the VC dimension h. Therefore, we can reduce the future error if we can reduce $h$ and also reduce the training error. In fact, SVM learning algorithms gives maximum margin function is one such function. The actual fault on the outlook data is bounded by a total of two terms is proved. The support vector machines (SVM) technique obtained the best levels in metrics such as precision (97\%). Thus, the well founded algorithm is SVM [33]-[39].

Advantages:

- Perform solid mathematic foundation (numerical calculation)

- Concept of structural risk minimization (minimization of wrong classification).

- Availability of vigorous tool and the algorithm to obtain the solution sooner and efficient.

- Perform regression analysis.

- Reduce the training error and leaves data for the future correction.

\section{Data set}

From our proposed system, the diagnosed results generated by the system corresponding to those patients have been saved in the database. The data are collected from real time database from UCI repository. In the 76 raw attributes with 603 records in the database, only 14 of them are usually used below we have lists the attributes. The model for calculating the efficiency of the proposed system uses the resultant data thus formed.

List of Attributes were as follows:

Table 5: Attributes of Heart Disease Dataset

\begin{tabular}{|c|c|c|c|}
\hline S.no & $\begin{array}{l}\text { Attribute } \\
\text { name }\end{array}$ & Type & DESCRIPTION \\
\hline 1 & Period & $\begin{array}{l}\text { Con- } \\
\text { tinu- } \\
\text { ous }\end{array}$ & Age of the patients in years \\
\hline 2 & $\begin{array}{l}\text { Sexual cate- } \\
\text { gory }\end{array}$ & $\begin{array}{l}\text { Dis- } \\
\text { crete }\end{array}$ & $\begin{array}{l}\text { Gender of the patients }(1=\text { male; } \\
0=\text { female })\end{array}$ \\
\hline 3 & $\begin{array}{l}\text { Chest Pain } \\
\text { Type }\end{array}$ & $\begin{array}{l}\text { Dis- } \\
\text { crete }\end{array}$ & $\begin{array}{c}\text { Defines the kind of chest pain } \\
\text { 1.Typical Angina } \\
\text { 2.Atypical Angina } \\
\text { 3.Non-Anginal Pain } \\
\text { 4.Asymptomatic }\end{array}$ \\
\hline 4 & $\begin{array}{l}\text { Trest Blood } \\
\text { Pressure }\end{array}$ & $\begin{array}{l}\text { Con- } \\
\text { tinu- } \\
\text { ous }\end{array}$ & $\begin{array}{l}\text { Inactive blood pressure (in } \mathrm{mm} \mathrm{Hg} \\
\text { on permission to the hospital) }\end{array}$ \\
\hline 5 & Cholesterol & $\begin{array}{l}\text { Con- } \\
\text { tinu- } \\
\text { ous }\end{array}$ & Serum cholesterol in $\mathrm{mg} / \mathrm{dl}$ \\
\hline 6 & $\begin{array}{l}\text { Fasting } \\
\text { Blood } \\
\text { Sugar }\end{array}$ & $\begin{array}{l}\text { Dis- } \\
\text { crete }\end{array}$ & $\begin{array}{l}\text { Fasting blood sugar }>120 \mathrm{mg} / \mathrm{dl} \\
\qquad(1=\text { correct; } 0=\text { false })\end{array}$ \\
\hline 7 & RestECG & $\begin{array}{l}\text { Dis- } \\
\text { crete }\end{array}$ & $\begin{array}{l}\text { Inactive electrocardiographic } \\
\text { results }(0=\text { normal; } 1=\text { have ST-T; } \\
2=\text { hypertrophy) }\end{array}$ \\
\hline 8 & Thalach & $\begin{array}{l}\text { Con- } \\
\text { tinu- } \\
\text { ous }\end{array}$ & Utmost heart rate achieved \\
\hline
\end{tabular}

\subsection{Support vector machines (SVM)}




\begin{tabular}{|c|c|c|c|}
\hline 9 & Exang & $\begin{array}{l}\text { Dis- } \\
\text { crete }\end{array}$ & $\begin{array}{l}\text { Apply induced angina }(1=y e s ; \\
0=\text { no })\end{array}$ \\
\hline 10 & Old peak & $\begin{array}{l}\text { Con- } \\
\text { tinu- } \\
\text { ous }\end{array}$ & $\begin{array}{l}\text { ST depression induced by exercise } \\
\text { relative to rest }\end{array}$ \\
\hline 11 & Slope & $\begin{array}{l}\text { Dis- } \\
\text { crete }\end{array}$ & $\begin{array}{l}\text { The slope of the crest exercise ST } \\
\text { segment }(1=\text { up sloping; } 2=\text { flat slop- } \\
\text { ing; } 3=\text { down sloping) }\end{array}$ \\
\hline 12 & $\mathrm{CA}$ & $\begin{array}{l}\text { Dis- } \\
\text { crete }\end{array}$ & $\begin{array}{c}\text { Number of key vessels }(0-3) \\
\text { decorated by fluoroscopy }\end{array}$ \\
\hline 13 & Thal & $\begin{array}{l}\text { Dis- } \\
\text { crete }\end{array}$ & $\begin{array}{c}3=\text { usual } ; 6=\text { permanent fault; } \\
7=\text { reversible fault }\end{array}$ \\
\hline 14 & $\begin{array}{l}\text { Concept } \\
\text { class } \\
\text { \{or }\} \\
\text { Num }\end{array}$ & $\begin{array}{l}\text { Dis- } \\
\text { crete }\end{array}$ & $\begin{array}{c}\text { The predicted attribute-judgment } \\
\text { of heart bug (angiographic disease } \\
\text { status)(value } 0=<50 \% \text { diameter nar- } \\
\text { row ; value } 1=>50 \% \\
\text { diameter narrow) }\end{array}$ \\
\hline
\end{tabular}

\section{Result and discussion}

Several Data Mining techniques were compared among their best results obtained from the method (algorithm) called support vector machines, based on the metrics, Precision $97.70 \%$, TpRate $97.70 \%$, FpRate $0.02 \%$, and Recall $97.70 \%$. From those results, it is clearly show that the propose method has a peak percentage in the appraise metrics which has testimonial to indicate that it is an efficient and exact method for the diagnosis of cardiovascular diseases, improving the outcomes obtained in previous studies.

\section{Performance analysis}

In data mining, the performance analyses of various algorithms are shown. In analysis, The Support Vector Machine (SVM) shows that, it has more (accuracy) prediction rate and less false rate. From the below bar chart(7), it is clearly visible that Naive Bayes algorithm shows precision rate $(95 \%)$ and also has false rate $(0.54 \%)$. Then the algorithm $\mathrm{J} 48$ shows precision rate (96\%) and also has false rate $(0.34 \%)$. Then Lazy IBK algorithm shows precision rate $(97 \%)$ and also has false rate $(0.32 \%)$. Lastly, the SVM algorithm shows precision rate $(97.70 \%)$ and also has false rate $(0.02 \%)$ which has the highest precision rate and also has lowest false rate comparing to all other algorithms. So, SVM is the effective algorithm to predict the heart attack in early stages.

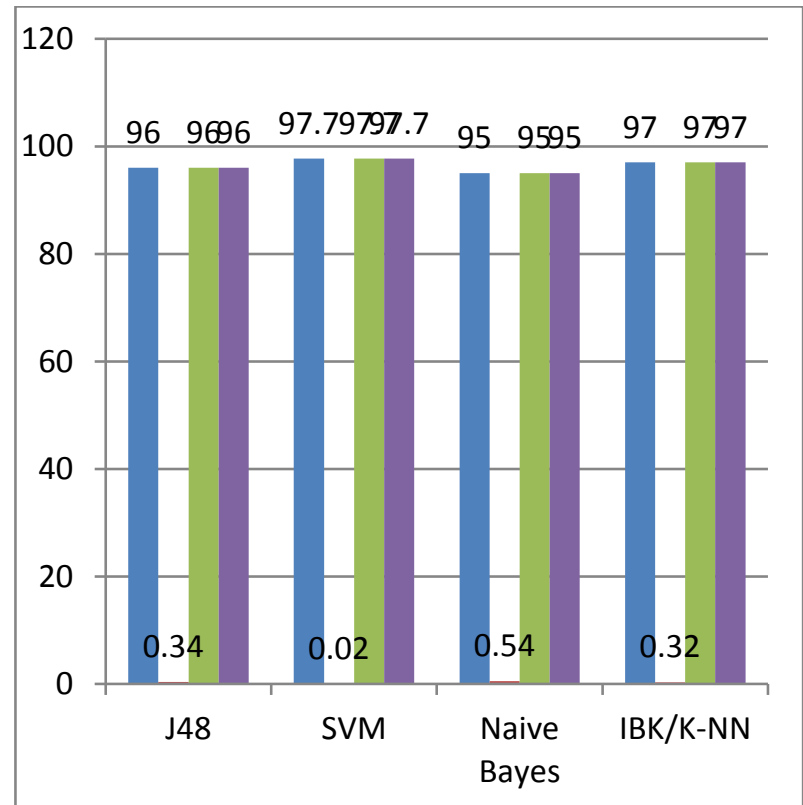

Fig. 1: True Positive Rate, True False Rate, Prediction Rate and Recall of Various Data Mining Algorithms.

Blue represents TPositive Rate

Red represents TFalse Rate
Green represents Prediction

Purple represents Recall

Chart 7: True Positive Rate, True False Rate, Prediction Rate and Recall of Various Data Mining Algorithms

Where,

TP

Precision $=\mathrm{TP}+\mathrm{FP}$

TPTpRate $=$ TP + FN

FP

FpRate $=\mathrm{FP}+\mathrm{VN}$

VP

Recall $=\mathrm{VP}+\mathrm{VN}$

Represents,

$\mathrm{TP}=$ True Positive

$\mathrm{FP}=$ False Positive

$\mathrm{FN}=$ False Negative

$\mathrm{FP}=$ False Positive

$\mathrm{VN}=$ Verdaderos Negative

$\mathrm{VP}=$ Verdaderos Positive

\section{Conclusion and future enhancement:}

Major life threatening disease that leads to death is Heart disease. Heart is the most essential organ of the human body as life is dependent on proficient working of heart. In order to save a patient's life, the doctors and medical examiners are being taking lots of efforts. The consultant of doctor's determination can make without the advice of specialists because of the software develop by the advancement in computer technology. By the way, after analyzing of all algorithms the highest precision rate will be Support Vector Machine (SVM).Data mining will play a vital role in medical areas especially use for prediction.

\section{References}

[1] Ajad Patel, Sonali Gandhi, Swetha Shetty,Prof. Bhanu Tekwani.(2017). Heart Disease Prediction Using Data Mining, 4(1):1705-1707.

[2] Akshaya, Nivetha Priya.M., Ms. Kartika's. (2015). Data Mining Techniques to Diagnose Heart Diseases, 2(4):132-134.

[3] Dr. P. C. Agrawal and Aparna Chaturvedi.(2015).An Analytical Study of Data Mining with the Help of UML Model and its Application,4(3):87-90.

[4] Era Singh Kajal and Ms. Nishika.(2016).Prediction of Heart Disease using Data Mining Techniques,2(3):1-7.

[5] Fabio Mendoza Palechor*,Alexis De la Hoz Manotas,Paola Ariza Colpas, Jorge Sepulveda Ojeda, Roberto Morales Ortega and Marlon Piñeres Melo.(2016) Cardiovascular Disease Analysis Using Supervised and Unsupervised Data Mining Techniques, 12(2):81-90 .

[6] H K Shifali, Dr. B. Srinivasu, Rajashekar Shastry and B N Ranga Swamy. (2017). Mining of Medical Data to Identify Risk Factors of Heart Disease Using Frequent Itemset, 4(1):1613-1619.

[7] K.Manimekalai.(2016). A Proficient Heart Disease Prediction Method Using Different Data Mining Tools, 6(3):2676-2680.

[8] P.Santhi, V.Murali Bhaskaran "Performance of Clustering Algorithms in Healthcare Database", International Journal for Advances in Computer Science, Volume 2, Issue 1, March 2010

[9] Megha Shahi and Er. Rupinder Kaur Gurm. (2017). Heart Disease Prediction System Using Data Mining Techniques - A Review, 3(4):73-77.

[10] Prof. Mamta Sharma, Farheen Khan, Vishnupriya Ravichandran. (2017). Comparing Data Mining Techniques Used For Heart Disease Prediction, 4(6):1161-1167.

[11] S.Keerthi, S.Dhivya, Comparison of RVM and SVMClassifier Performance in Analysing the Tuberclosis in Chest X Ray. Vol.10, No.36 Sep (2017). 0974-5572. 
[12] Rani Geetika. (2016).Hierarchical Clustering Techniques In Data Mining: A Comparative Study, 2(1):31-36.

[13] R. Thanigaivel and K. Ramesh Kumar. (2016).Boosted Apriori: an Effective Data Mining Association Rules for Heart Disease Prediction System, 24(1):192-200.

[14] Sharan Monica L and Sathees Kumar B. (2016).Analysis of Cardiovascular Heart Disease Prediction Using Data Mining Techniques, 4(1):55-58.

[15] Shashikant Ghumbre, Chetan Patil, and Ashok Ghatol. (2011).Heart Disease Diagnosis using Support Vector Machine, 84-88.

[16] Sonam Nikhar and A. M. Karandikar. (2016). Prediction of Heart Disease Using Data Mining Techniques - A Review, 3(2):1291-1293.

[17] Ms S. Vijayarani and Ms M. Muthulakshmi (2013).Comparative Analysis of Bayes and Lazy Classification Algorithms, 2(8):31183124.

[18] Xindong Wu · Vipin Kumar · J. Ross Quinlan · Joydeep Ghosh · Qiang Yang · Hiroshi Motoda offrey J. McLachlan · Angus Ng · Bing Liu · Philip S. Yu ·Zhi-Hua Zhou · Michael Steinbach · David J. Hand and Dan Steinberg. (2007). Top 10 algorithms in data mining, 14:1-37.

[19] Dr.P.Santhi, K.Deepa, Classification System for Identifying the chemical Structure Using the Support Vector Machine. Vol.03, No.1, June (2017). 2349-7866.

[20] S.Thilagamani, N. Shanthi," Object Recognition Based on Image Segmentation and Clustering", Journal of Computer Science,Vol. 7,No.11,pp. 1741-1748, 2011.

[21] S.Thilagamani, N. Shanthi," Gaussian and gabor filter approach for object segmentation", Journal of Computing and Information Science in Engineering,Vol.14,Issue 2, pp. 021006,2014.

[22] Dr.P.Santhi, S.Kiruthika," Lung Based Disease prediction Using Lobe Segmentation Based on Neural Networks", International Journal of Pure and Applied Mathematics", Vol.118, No.8, PP. 499-504 2018 .

[23] P.Santhi, R.Vikram," Implementation Of Classification System Using Density Clustering Based Gray Level Co Occurrence Matrix (DGLCM) For Green Bio Technology", International Journal of Pure and Applied Mathematics",Vol.118, No.8,PP. 191-195,2018.

[24] S.Thilagamani, V.Manochitra,"An Intelligent Region-Based Method for Detecting Objects from Natural Images", International Journal of Pure and Applied Mathematics", Vol.118, No.8, PP.473-478, 2018.

[25] S. Thilagamani, N.Shanthi, "A Novel Recursive Clustering Algorithm for Image Oversegmentation”, European Journal of Scientific Research, Vol.52, No.3, pp.430-436, 2011.

[26] S.Thilagamani and S. Uma Mageshwari," Risk appraisal for cardiovascular disease among selected young adult women in Coimbatore, India", Indian Journal of Science and Technology, Vol. 3, No. 6 ,PP.672-675,2010

[27] T. Mekala, P. Nandhini," Modified Agglomerative Clustering for Web Users Navigation Behavior", International Journal of Advanced Networking and Applications, Vol. 05, Issue: 01, PP.1842-1846, 2013.

[28] S.Thilagamani,N. Shanthi, "Literature survey on enhancing cluster quality", International Journal on Computer Science and Engineering Vol. 02, No. 06, pp1999-2002, 2010.

[29] K.Deepa, Y.Naveen Raj, "Enhancing the Performance in WSN using Distributed Tracking Algorithm", International Journal of Pure and Applied Mathematics Vol. 118, No. 9 2018, 717-722.

[30] G.Ranjith , M.Vinoth, "An Unified Approach for Effective Use of Cloud Metering Service", International Journal of Pure and Applied Mathematics Vol. 118, No. 9 2018, 801-806.

[31] E.T.Venkatesh,P.Tangaraj, S. Chitra, "Classification of cancer gene expressions from micro-array analysis", International Conference Innovative Computing Technologies (ICICT), 2010

[32] V.Baby Deepa, P.Thangaraj, S.Chitra," Investigating principal component analysis for classification of EEG data", International Conference on Networking and Information Technology (ICNIT), PP.461-464, 2010.

[33] Hema.C.R, Paulraj.M.P \& Ramkumar.S, "Classification of Eye Movements Using Electrooculography and Neural Networks”, International Journal of Human Computer Interaction, Vol.5 (4), pp.5163, 2014.

[34] Hema, C. R., Ramkumar, S., \& Paulraj, M. P. , "Idendifying Eye Movements using Neural Networks for Human Computer Interaction", International Journal of Computer Applications, 105(8), pp 18 26, 2014.

[35] S.Ramkumar, K.SatheshKumar, G.Emayavaramban, "EOG Signal Classification Using Neural Network for Human Computer Interaction", International Journal of Computer Theory and Applications, Vol.9(24), pp.223-231, 2016
[36] Ramkumar, Dr.K.Satheshkumar and G.Emayavaramban" Nine States HCI using Electrooculogram and Neural Networks", IJET, Vol. 8(6), pp. 3056-3064, Jan 2017.

[37] S.Ramkumar, K.Sathesh Kumar G.Emayavaramban," A Feasibility Study on Eye Movements Using Electrooculogram Based HCI' IEEE- International Conference on Intelligent Sustainable Systems, pp.384-388, Dec-2017.

[38] G.Emayavaramban, S.Ramkumar, A.Amudha and K.Sathesh Kumar "Classification Of Hand Gestures Using FFNN And TDNN Networks", International Journal of Pure And Applied Mathematics, Vol.118 (8) Pp. 27-32, Jan 2018.

[39] S.Ramkumar, K.Sathesh Kumar, T.Dhiliphan Rajkumar, M.Ilayaraja, K.Shankar, "A review-classification of electrooculogram based human computer interfaces", Biomedical Research, 29(6), Pp. 10781084, April 2018. 